\title{
A RELEVÂNCIA DAS ATIVIDADES DO IEEE WIE UFJF PARA DESENVOLVIMENTO DE SOFT SKILLS EM GRADUANDAS EM ENGENHARIA
}

DOI: 10.37702/2175-957X.COBENGE.2021.3596

Camila Costa Rocha - camila.rocha@engenharia.ufjf.br

UFJF

Rua Rodrigues Alves 1160

35040-440 - Governador Valadares - MG

Franciane da Silva Pereira - franciane.pereira@engenharia.ufjf.br

UFJF

Rua Geraldinho Barbosa 1693

35041-820 - Governador Valadares - MG

Alissa Oliveira Reis - aliolireis@gmail.com

Universidade Dederal de Juiz de Fora

Rua João Teixeira Lopes Filho 190

36060-030 - Juiz de Fora - MG

Rafaela Oliveira dos Anjos - rafaela.anjos@engenharia.ufjf.br Universidade Federal de Juiz de Fora

RUA ARISTÓTELES BRAGA 345

36037-010 - Juiz de Fora - MG

Mariana Guedes Mattos - mariana.mattos@engenharia.ufjf.br Universidade Federal de Juiz de Fora

Rua Domingos Húngaro 4800

36033-550 - Juiz de Fora - MG

Mariana Larissa Antunes da Costa - mariana.antunes@engenharia.ufff.br Universidade Federal de Juiz de Fora

Praca do Cruzeiro 42

36016-260 - Juiz de Fora - MG

Janaina Goncalves de Oliveira - janaina.oliveira@ufjf.edu.br UFJF

Avenida presidente Costa e Silva 2391 


\section{(C. COBENGE

36037-000 - Juiz de Fora - MG

Luis Henrique Lopes Lima - luishenrique.lima@ufjf.edu.br Universidade Federal de Juiz de Fora

Rua José de Castro Barbosa 84

36033-680 - Juiz de Fora - MG

Resumo: O desenvolvimento de soft skills está em pauta dentro do mercado de trabalho visto que muitos profissionais possuem capacidades técnicas mas não sabem se comunicar ou ter empatia de uma forma assertiva. As capacidades técnicas são imprescindíveis para qualquer profissional, entretanto elas podem ser facilmente desenvolvidas com devidos treinamentos e gestão do conhecimento, ao contrário das competências interpessoais. Por isso, o IEEE Women in Engineering UFJF, um grupo de afinidade estudantil do IEEE, faz acontecer atividades para que suas voluntárias consigam desenvolver capacidades interpessoais e conseguir, com maior facilidade, vagas no mercado de trabalho.

Palavras-chave: Soft skills. Mulheres em Engenharia. Empoderamento. STEM 


\section{A RELEVÂNCIA DAS ATIVIDADES DO IEEE WIE UFJF PARA $O$ DESENVOLVIMENTO DE SOFT SKILLS EM GRADUANDAS EM ENGENHARIA}

\section{INTRODUÇÃO}

$\mathrm{Na}$ atual realidade dos recém-formados dos cursos de engenharia, é comprovado que somente $10 \%$ dessa parcela possui a capacidade de efetivamente entrar no mercado de trabalho (REDDY, 2013). Esse dado, se faz verdade, pelo fato de que apenas as capacidades técnicas não são mais significado de bons profissionais, devidamente preparados para o mercado de trabalho global. Hoje é esperado e se faz necessário que os engenheiros disponham de uma aptidão para comunicação, juntamente com outras competências transversais, conhecidas como soft skills. Essas habilidades não somente qualificam para a entrada no mercado de trabalho como também servem de facilitadoras para um bom desempenho ao longo da própria graduação, posto que tais qualificações estão atreladas a relações interpessoais e mesmo durante a formação, os estudantes de engenharia vivenciam tais relações.

A partir desse cenário, a graduanda em engenharia deve buscar aprimorar essas aptidões. Pensando nisso, o grupo IEEE WIE (Women in Engineering, em tradução literal Mulheres na Engenharia) da Universidade Federal de Juiz de Fora, através da atuação dos membros nos projetos desenvolvidos, tem como um dos objetivos o desenvolvimento de habilidades que não são tão bem trabalhadas ao longo do curso de graduação.

Este trabalho pretende apresentar como as voluntárias do IEEE WIE UFJF podem e conseguem desenvolver essas competências através da realização dos projetos propostos pelo grupo. Ele se divide em seções que contextualizam o Instituto de Engenheiros Eletricistas e Eletrônicos (IEEE) com o IEEE WIE UFJF e o desenvolvimento de habilidades em graduandas dos cursos de engenharia.

\section{INSTITUTO DE ENGENHEIROS ELETRICISTAS E ELETRÔNICOS (IEEE) E O IEEE WOMEN IN ENGINEERING (WIE)}

Criado em 1963 nos Estados Unidos e conhecido por ser a maior organização técnica e profissional sem fins lucrativos do mundo, congregando mais de 416 mil associados, dos quais aproximadamente 120 mil são estudantes, em cerca de 160 países e com inúmeras contribuições em várias áreas da ciência, o Institute of Electrical and Electronics Engineers (IEEE - Instituto dos Engenheiros Eletricistas e Eletrônicos) tem como principal objetivo colaborar no incremento da prosperidade mundial mediante a inovação tecnológica no que se refere a eletricidade e informação, sempre em prol da humanidade. O instituto cumpre esse propósito por meio da realização de congressos, criação de padrões e normas, além de ser o provedor de mais de $30 \%$ das publicações científicas na área de Engenharia Elétrica, Computação e Tecnologia.

Trabalhando junto a essa organização, o IEEE Women in Engineering (WIE) tem como principal objetivo promover engenheiras e cientistas, além de inspirar meninas do mundo todo a seguirem seus interesses acadêmicos em engenharia e ciência. Sua rede global conecta cerca de 20.000 membros em mais de 100 países, com isso, os membros conseguem formar uma rede mundial de conhecimento, criar amizades para a vida toda, adquirem mentores influentes e fazem a diferença em benefício da humanidade. 


\subsection{Ramo Estudantil IEEE UFJF}

As ações do IEEE são divididas em 10 regiões ao longo do globo. As regiões se subdividem em Seções, que servem como centros de atividade profissional para engenheiros vinculados da organização, além das filiais estudantis nas instituições de ensino, os chamados Ramos Estudantis. Dentre as 333 Seções do IEEE existentes em todo o mundo, 5 se encontram no Brasil e juntas formam o Conselho Brasil do IEEE, no qual o Ramo Estudantil IEEE UFJF se encontra, sendo pertencente à Seção Minas Gerais.

O núcleo atua na disseminação de tecnologia desde 1991, tendo em sua criação a finalidade de colaborar na implementação de cursos de pós-graduação em Engenharia Elétrica na Universidade, visto que por meio dele seria possível um fácil acesso à atualizadas publicações científicas de melhor qualidade, através de periódicos do IEEE adquiridos por intermédio do Ramo Estudantil a preços mínimos.

Atualmente, o Ramo Estudantil IEEE UFJF atua executando e desenvolvendo múltiplos projetos de cunho técnico, humanitário e/ou educacional, servindo de atividades complementares a formação dos engenheiros da universidade, já que englobam competências que não estão associadas às salas de aula, como é o caso do desenvolvimento das Soft Skills.

\subsection{O Grupo IEEE WIE UFJF}

O IEEE Women in Engineering (WIE) UFJF é um grupo de afinidade estudantil filiado ao IEEE WIE que deu início a suas atividades em 2004, sendo o primeiro grupo IEEE WIE estudantil brasileiro. O núcleo realiza seus projetos inspirados nos 17 Objetivos de Desenvolvimento Sustentável (ODS) da ONU, focado no $5^{\circ}$ - Igualdade de Gênero, sejam educacionais, técnicos e/ou humanitários em parceria com outros Grupos de Afinidades, Capítulos Técnicos e Segmentos Estudantis tanto do Ramo Estudantil IEEE UFJF quanto de outros lugares, dessa forma tanto a comunidade acadêmica e externa à UFJF é impactada. O grupo possui uma hierarquia de cargos por questões formais que se dividem entre Conselho, o qual possui presidente, vice-presidente, secretária, tesoureira e webmaster, líderes de projeto e apoios dos projetos, além da professora orientadora e outros professores parceiros

O grupo possui o objetivo de ajudar e incentivar a todas as meninas que queiram seguir ou a prosseguir nas áreas de STEM (Ciência, Tecnologia, Engenharia e Matemática, em português), além de criar uma rede de mulheres e homens que trabalham juntos, usando seus diversos conhecimentos, para inovar em benefício da humanidade. Atualmente conta com 21 voluntárias, mas já houve a participação de voluntários em anos anteriores. Com suas atividades já atingiu mais de 30 mil pessoas desde 2019. Em 2020, o grupo foi considerado exemplar a nível Brasil e América Latina e Caribe pelas suas atividades desenvolvidas. Além disso, o IEEE WIE UFJF também tem como propósito desenvolver as competências não exploradas na grade curricular dos membros, mas que são essenciais para sucesso no mercado de trabalho, seja indústria, seja academia.

\section{RELAÇÕES ENTRE AS SOFT SKILLS E O MERCADO DE TRABALHO}

O mundo atual não é o mesmo de anos atrás, as profissões se inseriram cada vez mais no contexto atual da revolução tecnológica. Embora não seja tão notável, as revoluções não se limitam apenas ao uso de novas tecnologias, mas também a forma como nos relacionamos e somos vistos no mercado de trabalho. Como exemplo, os cursos de 
datilografia poderiam ser diferenciais nos tempos passados, porém hoje em dia, os diferenciais para o mercado de trabalho se encontram numa camada mais profunda.

Um contexto histórico pode ser oferecido nessa situação, no livro "Soft skills: competências essenciais para os novos tempos" escrito por Lucedile Antunes onde podemos encontrar o porquê de Hard Skills terem sido desenvolvidas mais cedo pela humanidade. Segundo a autora, por razões biológicas a mais de 300 mil anos quando os Homo Sapiens surgiram, as condições impostas a eles fizeram com que o desenvolvimento de características que reduzissem as ameaças daquele momento fosse desenvolvido primeiro. As soft skills podem ser definidas segundo José Carlos Martins autor do livro "Soft Skills: conheça as ferramentas para adquirir, consolidar e compartilhar conhecimentos", como comportamentos e competências inatas ou aperfeiçoadas do indivíduo, as quais não se aprendem em uma escola. Além disso, trata-se de competências que agregam uma melhor produtividade podendo facilitar a comunicação no ambiente de trabalho.

Do ponto de vista do mercado de trabalho já existem comprovações de que profissionais que possuem as Soft Skills conseguem obter os melhores resultados em suas atividades. No texto "Soft skills training boosts productivity" (em português "O treinamento de habilidades pessoais aumenta a produtividade"), disponibilizado no site da Universidade de Michigan, podemos ver o resultado obtido pela professora assistente de economia empresarial e políticas públicas, Achyuta Adhvaryu. O trabalho mostra que trabalhadores de fábricas na Índia que foram apresentados às Soft Skills se mostraram $12 \%$ mais produtivos do que os outros. O World Economic Forum elencou em um TOP 10 Soft Skills que serão de extrema importância para o mercado de trabalho, dentre elas encontramos: Inteligência emocional, criatividade, pensamento crítico e solução de problemas. Voltando seis anos atrás podemos fazer um comparativo entre as Soft Skills que estavam em foco e as atuais, conforme figura 1. Observando o primeiro lugar, encontramos a resolução de problemas, porém o pensamento crítico superou a colaboração com outros. É possível concluir olhando as demais posições que as Soft Skills não são absolutas. É possível afirmar ainda, que o surgimento de novas habilidades se deve ao fato da constante mudança de necessidades pessoais com o passar do tempo. A criatividade poderia ser um requisito não tão almejado a alguns anos, porém atualmente se torna extremamente necessária para que possamos atingir a inovação. Diante disso, percebe-se a necessidade crescente do desenvolvimento, ainda nas instituições de ensino superior, de conhecimento e aprimoramento pessoal.

Figura 1 - Ranking das Soft Skills

\section{TOP 10 SKILLS}

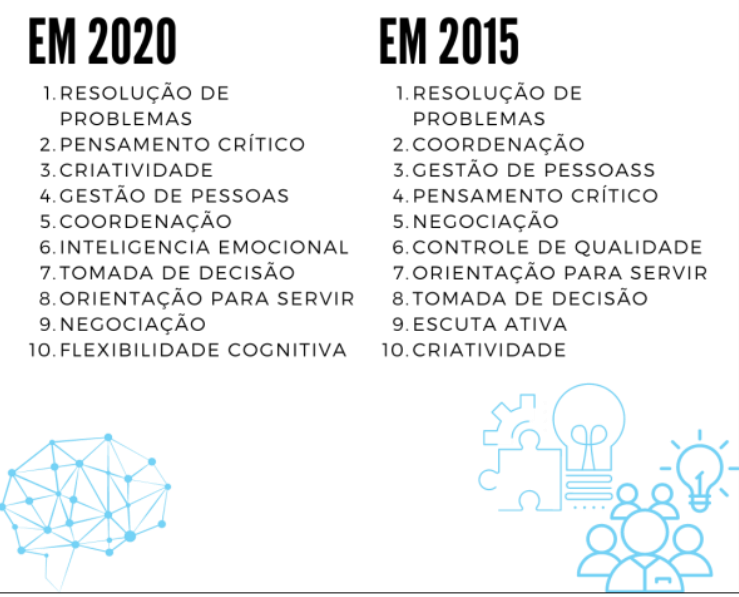

Fonte: Future of Jobs Report, World Economic Forum (adaptada). 


\section{OS PROJETOS DESENVOLVIDOS PELO IEEE WIE UFJF}

Conforme dito anteriormente, os projetos do IEEE WIE UFJF estão alinhados aos valores do IEEE de difundir a tecnologia e a inovação em benefício da humanidade, e aos do IEEE WIE, de facilitar e colaborar para o desenvolvimento das voluntárias em áreas ligadas ao STEM. Além disso, sempre estão atrelados aos 17 ODSs da ONU, podendo ser citados o 4 - Educação de Qualidade, 5 - Igualdade de Gênero, 10 - Redução das Desigualdades e 17 - Parcerias e Meios de Implementação. Cada projeto possui uma líder, a qual tem a responsabilidade de, junto aos voluntários, realizar as atividades. Faz-se possível ressaltar a importância destes projetos para deter a evasão feminina nos cursos de STEM e gerar uma cadeia de apoio dentro da instituição a fim de atender aos momentos necessários, ou seja, antes e durante o ingresso à faculdade. Atualmente o núcleo possui 5 projetos ativos, conforme descrito abaixo.

\subsection{Projeto Circuito Científico}

O Circuito Científico é um dos projetos do IEEE WIE UFJF que se tornaram Projeto de Extensão da UFJF. Atingindo os ODSs 5, 10 e 17, ele é realizado em parceria com o IEEE SIGHT UFJF, com intuito de levar um laboratório de ciências de baixo custo para as escolas da região de Juiz de Fora, a fim de que os alunos das mais diversas faixas etárias e econômicas tenham contato com a ciência usando materiais de fácil acesso. O objetivo principal do projeto é inserir no mundo científico e desmistificar a barreira existente sobre esse tipo de propriedade intelectual. Para que as atividades aconteçam, sempre ficam presentes um voluntário e uma voluntária, para já mostrar representatividade às meninas.

Inicialmente os responsáveis expõem os conceitos teóricos, e dão a oportunidade das crianças testarem, na prática, os experimentos, como: desenvolver seu próprio foguete com palito de churrasco, papel alumínio e fósforo, montar o próprio circuito elétrico com massinha de modelar e leds, observar as experiências com indicadores ácido-base e o líquido não newtoniano, com água e amido de milho.

Figura 2 - Projeto Circuito Científico
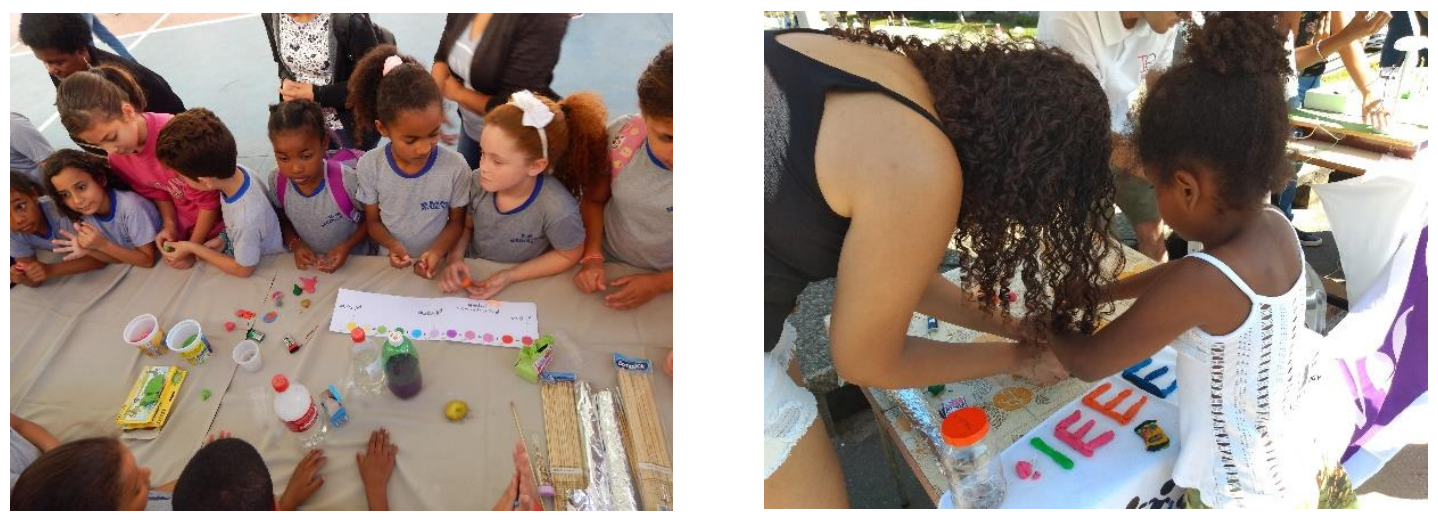

Fonte: Próprio autor 2021 


\subsection{Projeto Mutirão Tecnológico}

O Mutirão Tecnológico é outro Projeto de Extensão da UFJF, iniciado no IEEE WIE UFJF em 2018. Ele atende efetivamente os ODSs 5, 10 e 17. Seu objetivo é transmitir conhecimentos adquiridos na universidade, para a população, especialmente para mulheres em situação de vulnerabilidade social, gerando empoderamento através do conhecimento. As atividades consistem em workshops de Instalações Elétricas Básicas, com parte prática com tópicos como troca de resistência de chuveiro, o funcionamento de um disjuntor, a fabricação de uma extensão elétrica e de uma luminária, e teórica com dicas de economia de energia, medidas de segurança, como interpretar sua conta de luz e conceitos físicos, como tensão, corrente, resistência e potência. A abordagem é elaborada de acordo com as necessidades e interesses de cada público-alvo, além da expansão para o meio online, onde vídeos são publicados no YouTube e cartilhas com variados temas são compartilhados por meio de redes sociais do projeto.

Nesse momento, podemos ressaltar a importância de um projeto desse tipo, onde conceitos comumente vistos com alto grau de complexidade e associados erroneamente apenas ao gênero masculino são levados para a população de forma didática. Além disso, as barreiras existentes entre a parte da população que não tem acesso as instituições de ensino superior é aos poucos dissuadida, demonstrando que a universidade pública é um dos principais meios de transformação da sociedade.

Figura 3 - Projeto Mutirão Tecnológico
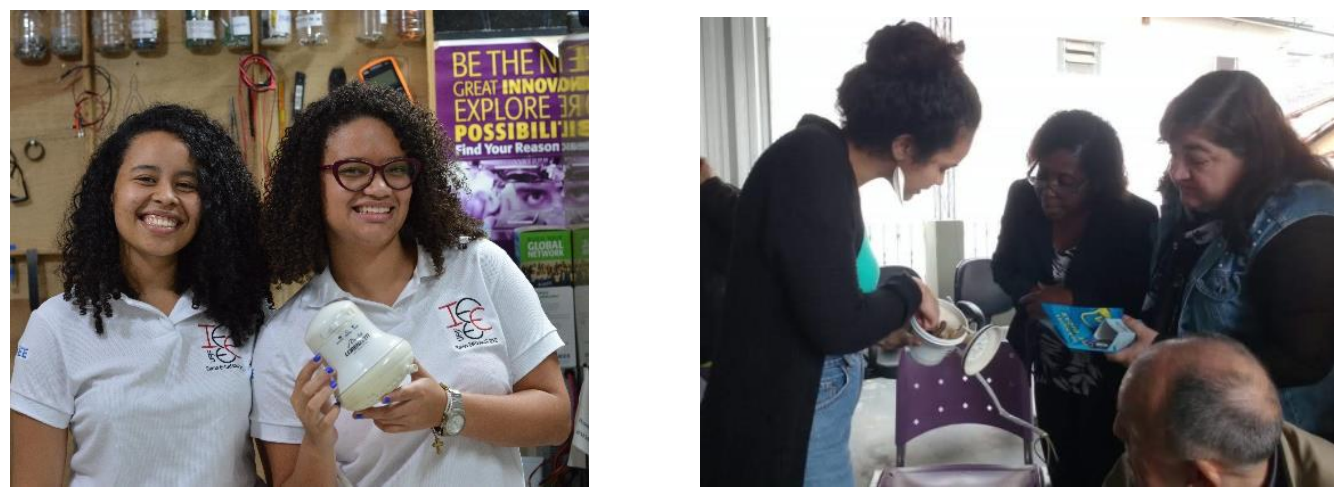

Fonte: Próprio autor 2021

\subsection{Projeto WIETech}

Os cursos nas áreas de conhecimento ligadas a ciências, tecnologia, engenharia e matemática possuem um viés histórico ligado à comunidade masculina. Essa desigualdade dentro dos cursos existe ainda atualmente, o que faz com que as mulheres que ingressam nesses cursos enfrentam preconceitos pré-existentes. Nesse contexto surge o WIETech, buscando diminuir não só a discrepância entre o ensino prático oferecido pela instituição de ensino como também acolher essa classe, ainda atingindo os objetivos 5 e 10 dos ODSs. O projeto propõe workshops para integrantes do IEEE WIE UFJF e a comunidade acadêmica. Atualmente algumas habilidades são indispensáveis para a inserção no mercado de trabalho, uma delas é o conhecimento em programação. Visando suprir essa necessidade o primeiro minicurso foi voltado para essa área, linguagens como $\mathrm{C}$, Python $\mathrm{e}$ $\mathrm{C}++$ foram abordadas. 
Como incentivo à inclusão, a segunda ação do projeto se resumiu em uma Oficina de LIBRAS (Língua Brasileira de Sinais), ministrado conjuntamente pela Faculdade de Letras da UFJF, trazendo à tona um tema que não é muito incentivado. Além disso, foi oferecido um workshop sobre o tema para a Faculdade de Engenharia, durante a Semana da Engenharia, realizada pelo Diretório Acadêmico, em janeiro.

Figura 4 - Projeto WIETech
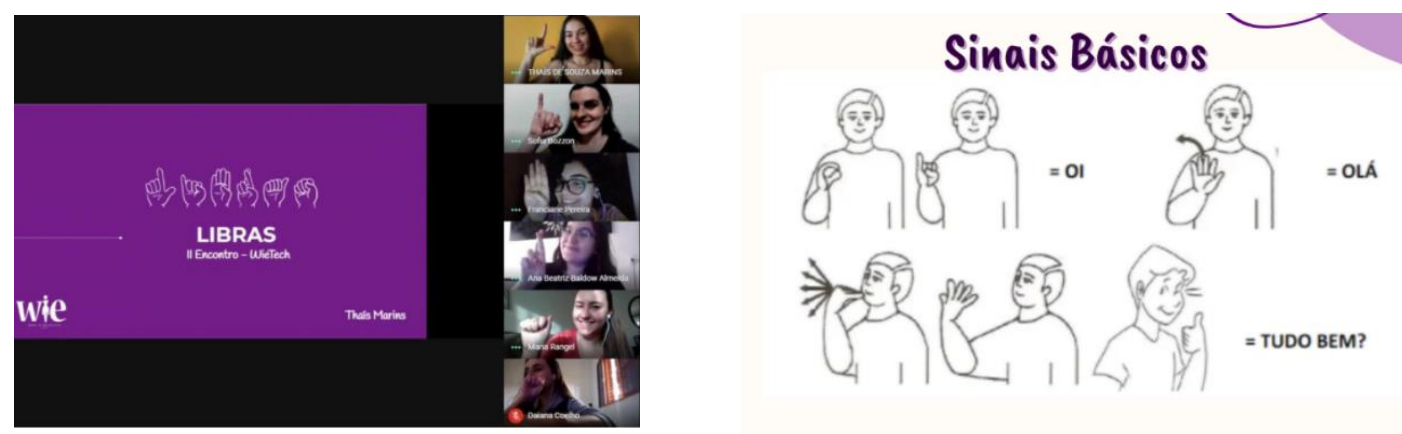

Fonte: Próprio autor 2021

\subsection{Projeto Motiva WIE}

O projeto Motiva WIE é um projeto que, como o próprio nome diz, pretende motivar mulheres nas áreas de STEM, e está alinhado ao ODS 5 - Igualdade de Gênero. Para tal, desenvolve atividades a fim de colaborar com a permanência e a inserção de mulheres nessas áreas. Seu principal projeto foi o Mamãe WIE, no qual o grupo foi atrás de mulheres que engravidaram durante a graduação e teve o objetivo de diminuir a evasão universitária de mulheres. O resultado do projeto foi um vídeo, o qual atingiu mais de 2900 visualizações no Facebook, e um ofício para a UFJF requisitando mais trocadores dentro dos banheiros da universidade. Outra atividade proposta pelo projeto e que já está em sua terceira edição é a Semana das Mulheres. Em 2019 e 2020, ocorreu de maneira presencial, já em 2021, devido ao cenário da pandemia, foi realizada remotamente. O objetivo dessa iniciativa é trazer mulheres para palestras, workshops e mesas redondas para falarem desde temas relacionados aos trabalhos delas quanto sobre mulheres em STEM, em geral.

Figura 5 - Projeto Motiva WIE
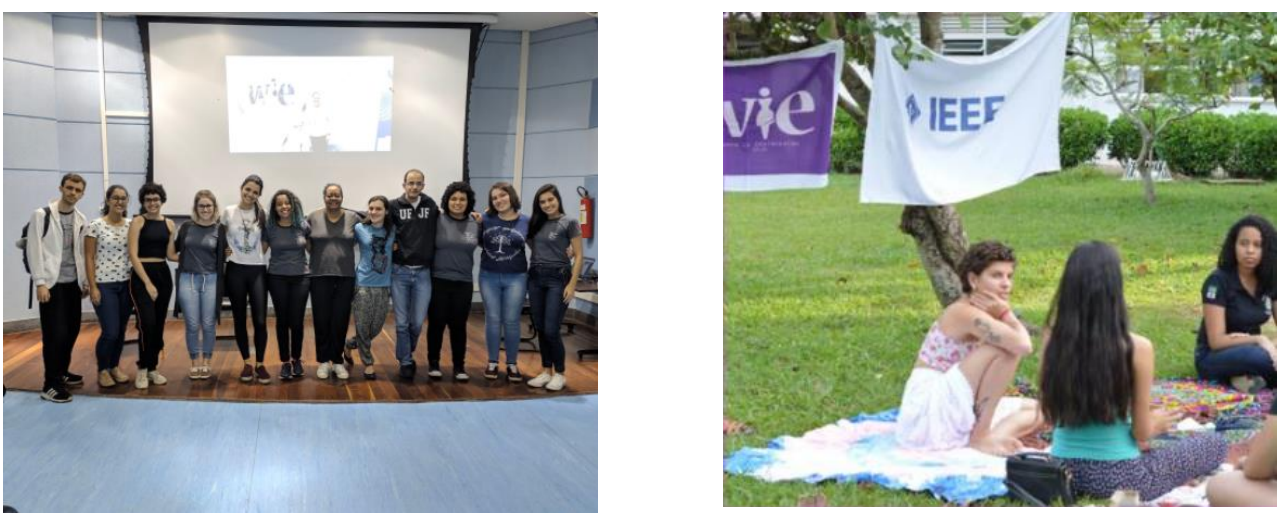

Fonte: Próprio autor 2021 


\subsection{Projeto de Engenheira para Futura Engenheira}

O projeto De Engenheira para Futura Engenheira visa, em suma, gerar empoderamento através da representatividade, consistindo, inicialmente na divulgação de fotos e textos de Engenheiras e graduandas de Engenharia nas redes sociais do IEEE WIE UFJF. Porém, em 2019, iniciou-se uma nova trajetória, trazendo mulheres das áreas de exatas para participar da iniciativa. As referidas têm a oportunidade de contar um pouco de suas histórias de vida, trajetórias, dificuldades no curso e dar dicas às graduandas e futuras graduandas. O projeto consegue colaborar ativamente com o ODS 5 - Igualdade de Gênero.

Figura 6 - Projeto de Engenheira para Futura engenheira
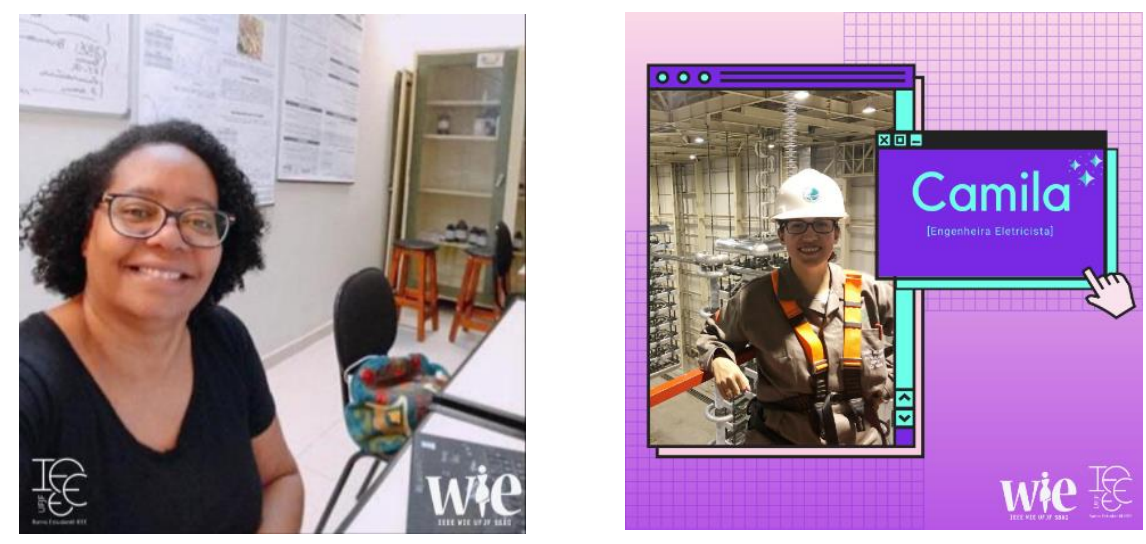

Fonte: Próprio autor 2021

\section{RELAÇÃO DOS PROJETOS E A JORNADA DO VOLUNTÁRIO}

Ao ingressar na faculdade geralmente os alunos se encontram fragilizados com a mudança completa de ambientes e realidades e nesse momento é importante ressaltar também que, em geral, o tema competências interpessoais ou soft skills às vezes ainda não é nem conhecido. Na maioria das vezes, o primeiro contato com essas competências vem quando o discente se inscreve para participar de alguma organização estudantil, sejam eles o Programas de Educação Tutorial, para quem geralmente tem interesses para seguir carreiras acadêmicas, ou Empresas Juniores, para quem geralmente tem interesses em ir para a indústria. Entretanto, a proposta do IEEE Women in Engineering consegue englobar essas duas perspectivas, ao ter projetos de cunho técnico e educacional.

A partir do momento que o voluntário adentra ao IEEE WIE UFJF, ele tem a oportunidade de participar ativamente de projetos, fazendo-os acontecer. Logo aí surge a oportunidade de desenvolver a competência proatividade. Ainda, é necessário pautar que cada projeto consiste em, no mínimo, três etapas, a saber: planejamento, comunicação e a aplicação. Para a primeira etapa, o pensamento criativo, a capacidade de treinamento e aprendizagem autônoma, para a segunda etapa, é necessário desenvolver a comunicação, visto que é necessário entrar em contato com pessoas que farão o grupo chegar até o público-alvo, seja a diretoria de uma escola, de outras organizações estudantis ou até mesmo empresas, para um possível patrocínio. Na etapa de aplicação, uma boa comunicação é mais imprescindível ainda, pois deve-se ter em mente que provavelmente terão que se comunicar de formas para cada público-alvo: crianças, universitários ou pessoas mais velhas. Ainda na última etapa são desenvolvidas competências como 
oratória, flexibilidade cognitiva, criatividade, resiliência e, se o projeto for de cunho humanitário, facilmente a capacidade de empatia é desenvolvida.

Pouco tempo depois que o voluntário entra em um projeto, é possível que, caso deseje, assuma a sua liderança. Para esse cargo já é necessário ter algumas das habilidades interpessoais desenvolvidas, pois, além delas, deverá ser capaz de delegar tarefas, uma forma de tomada de decisão, além de negociação, pois articula mais ativamente com os mediadores até o público-alvo e, como um bom líder, sempre deve estar disposto a ajudar e entender os seus liderados, levando novamente à empatia e à comunicação não-violenta. Ao fim da jornada como líder de projeto, a tendência é que a habilidade coordenação e inteligência emocional esteja bem desenvolvida. Depois de um certo tempo como líder de projeto, é possível que, por meio de eleição mediada pela Diretoria de Gestão de Pessoas, a voluntária se candidate à presidência do núcleo. Para esse cargo de grande importância e responsabilidade, essas soft skills serão ainda mais desenvolvidas, o que abre portas para diversas oportunidades, como falar sobre o núcleo em palestras, ter contato mais próximo com os professores orientadores e até mesmo representar o grupo em eventos maiores, o que ocorre com grande frequência.

Por fim, após sua jornada no IEEE WIE UFJF, em possibilidades de estágio, trainee, empregos ou editais para a carreira acadêmica, o voluntário, caso tenha aproveitado as oportunidades que o núcleo oferece e se dedique a elas, terá várias competências interpessoais desenvolvidas e terá mais chances de assertividade do que seus concorrentes. É válido ressaltar que não só as soft skills são importantes para o sucesso, o conhecimento técnico também é importante. Entretanto, as competências técnicas demandam muito menos tempo para serem desenvolvidas, visto que as interpessoais precisam de experiências reais, algo que pode se encontrar no IEEE WIE UFJF.

\section{CONSIDERAÇÕES FINAIS}

O IEEE WIE UFJF já é reconhecido nacional e internacionalmente pelos seus projetos. Em 2019 recebeu 4 prêmios nacionais, 1 a nível de América Latina e Caribe e 1 mundial e, em 2020, foram 6 a nível nacional, 1 a nível América Latina e Caribe e 2 a nível mundial. Além disso, como citado anteriormente, já atingiu mais de 30.000 pessoas, virtualmente e presencialmente. Faz-se relevante exprimir esses números pois eles são diretamente proporcionais ao esforço que as voluntárias de nível maior em hierarquia do grupo, fazem para que as voluntárias, em geral, tenham a melhor experiência no período que se dedicam ao grupo, principalmente em questão de desenvolvimento pessoal.

Em síntese, no final da gestão 2020, as próprias voluntárias elencaram algumas competências interpessoais que conseguiram desenvolver durante o ano, sendo elas: trabalho em equipe, oratória, autoconfiança, organização, responsabilidade, dedicação, comunicação, paciência, gestão de tempo, disciplina, empatia, delegação de tarefas e planejamento. Portanto, pode-se afirmar que as atividades promovidas pelo grupo são realmente efetivas para esse objetivo. Por fim, sugere-se que mais organizações estudantis valorizem e desenvolvam em seus voluntários tais competências.

\section{Agradecimentos}

Agradecemos a todas as voluntárias do IEEE WIE UFJF por fazerem esse projeto acontecer. Agradecemos também às professoras Janaína Oliveira Gonçalves e Zélia Ludwig e ao professor Luis Henrique Lopes Lima por todo apoio. Por fim, agradecemos a Faculdade de Engenharia da UFJF, a Pro Reitoria de Extensão da UFJF, a Seção Minas Gerais do IEEE e ao Conselho Brasil pelo suporte de sempre. 


\section{REFERÊNCIAS}

ANTUNES, Lucedile. Soft skills: competências essenciais para os novos tempos. Literary Books, 2020.

IEEE. History of IEEE. Disponível em: https://www.ieee.org/about/ieee-history.html. Acesso em: 23 Abr. 2021.

IEEE UFJF. Disponível em: https://edu.ieee.org/br-ufjf/grupos/sight/. Acesso em: 24 abr. 2021.

IEEE Women In Engineering UFJF - Ramo Estudantil IEEE UFJF, Ramo Estudantil IEEE UFJF. Disponível em: https://edu.ieee.org/br-ufjf/grupos/wie/. Acesso em: 24 Abr. 2021.

NAÇÕES UNIDAS BRASIL. Objetivos de Desenvolvimento Sustentáveis. Disponível em: https://brasil.un.org/pt-br/sdgs. Acesso em: 24 abr. 2021.

MARTINS, José Carlos Cordeiro. Soft Skills: conheça as ferramentas para você adquirir, consolidar e compartilhar conhecimentos. Brasport, 2017.

MICHIGAN NEWS. Soft skills training boosts productivity. Disponível em: <https://news.umich.edu/soft-skills-training-boosts-productivity/>. Acesso em: 23 abr. 2021.

REDDY, B. Balaji; GOPI, M. M.; ANEEL, Y. Having "Effective Communication Skills are Indispensable for the Successful Career of Engineering Students". Department of B\&HS, Siddharth Institute of Engineering \& Technology, Narayanavanam Road, Puttur-517583, Chittoor (Dist), AP (State), INDIA. IOSR Journal Of Humanities And Social Science (IOSRJHSS) Volume, v. 11, p. 20-22, 2013.

WIE. About IEEE WIE. Disponível em: https://wie.ieee.org/ Acesso em: 24 Abr. 2021.

WILLIAMS, Ray. The Growing Importance of "Soft Skills" in the Workplace. Medium, Disponível em: <https://raybwilliams.medium.com. Acesso em: 23 Abr. 2021.

\section{THE RELEVANCE OF IEEE WIE UFJF ACTIVITIES FOR THE DEVELOPMENT OF SOFT SKILLS IN GRADUATES IN ENGINEERING}

Abstract: The development of soft skills is on debate within the job market since many professionals have technical skills but don't know how to communicate or empathize in an assertive way to others. Technical skills are essential for any professional, however they can be easily developed with proper training and knowledge management, unlike interpersonal skills. For this reason, IEEE Women in Engineering UFJF, a student affinity group from IEEE, makes activities happen so that its volunteers can develop interpersonal skills and probably they will have greater opportunities in the job market in this regard.

Keywords: Soft skills. Women in Engineering. Empowerment. STEM 